\title{
UNSTABLE MORSE CODE RECOGNITION SYSTEM WITH EXPERT-GATING NEURAL NETWORK
}

\author{
DuU-Tong FuH AND CHING-Hsing LuO \\ Department of Electrical Engineering, \\ National Cheng Kung University, Tainan, Taiwan
}

ABSTRACT
The standard Morse code defines the tone ratio (dash/dot) and the silent ratio (dash-space/dot-
space) as 3:1. Since human typing ratio can't keep this ratio precisely and the two ratios --tone ratio
and silent ratio-- are not equal, the Morse code can't be recognized automatically. The requirement of
the standard ratio is difficult to satisfy even for an ordinary person. As for the unstable Morse code
typing pattern, the auto-recognition algorithms in the literature are not good enough in applications.
The disabled persons usually have difficulty in maintaining a stable typing speeds and typing ratios,
we therefore adopted an Expert-Gating neural network model to implement in single chip and recog-
nize online unstable Morse codes. Also, we used another method-- a linear back propagation recall-
ing algorithm, to implement in single chip and recognize unstable Morse codes. From three person
tests: Test one is a cerebral palsy; Test two is a beginner: Test three is a skilled expert, we have the re-
sults: in the experiment of test one, we have $91.15 \%$ (use 6 characters average time series as thresh-
olds) and $91.54 \%$ (learning 26 characters) online average recognition rate; test two have $95.77 \%$
and $96.15 \%$, and test three have $98.46 \%$ and $99.23 \%$ respectively. As for linear back propagation re-
calling method online recognized rate, we have the results from test one: $92.31 \%$ online average rec-
ognition rate; test two: $96.15 \%$ and test three $99.23 \%$ respectively. So, we concluded: The Expert-
Gating neural network and the linear back propagation recalling algorithm have successfully over-
come the difficulty of analyzing a severely online unstable Morse code time series and successfully
implement in single chip to recognize online unstable Morse code.
Biomed Eng Appl Basis Comm, 2002 (February): $14: 12$ - 19 .
Key word: Morse code, Expert-Gating neural network, back propagation, recognition.

\section{INTRODUCTION}

Within the last several years, the reduced keyboard, keystroke savings, and the use of Morse code [1-7] are providing effective alternative communication channels for individuals with physical limitations. In order to improve the life quality of the disables, we

Received: Nov. 16, 2001; accepted: Dec. 7, 2001

Correspondence:Ching-Hsing Luo, Professor

Department of Electrical Engineering

National Cheng Kung University

Tainan 70101, TAIWAN

E-mail: chluo@eembox.ee.ncku.edu.tw design a single switch keyboard with Morse code data transmission system for disabilities. This system will provide the disables with easy accesses to the computer appliances and therefore to the frontier of nonbarriers environment.

With single switch, Morse code samples a tonesilent signal with time series. Tone signals (switch down) can be a dot (short interval) or a dash (longer interval). Silent signal (switch up) can be a dot-space (short interval between dot and dash) or a dash-space (longer interval between character). The standard Morse code defines the tone ratio (dash/dot) and the silent ratio (dash-space/dot-space) as 3:1. Because human typing skill can't keep this ratio precisely and the two ratios --tone ratio and silent ratio-- are not equal, the Morse code can't be recognized automatically. The requirement of the standard ratio is difficult to satisfy 
even for an ordinary person. We already knew that Morse code auto-recognition could be very successful for the stable typing speeds and stable typing ratio of dash to dot intervals. As for the unstable Morse code typing pattern, the auto-recognition algorithms in the literature are not good enough in applications. From the real world situation that the disabled persons usually have difficulty in maintaining stable typing speeds and typing ratio, we therefore adopted an ExpertGating neural network model to recognize unstable Morse codes.

In the report of Luo \& Shih [4], a single switch Morse code automatic recognition system has been constructed. They have successfully used an adaptive unstable-speed prediction algorithm to deal with this problem. A fixed ratio was used in this algorithm. Therefore it has difficulty in recognizing an unstableratio signal. Although Shih \& Luo [5] have successfully designed a LMS and matching algorithm to raise the recognition rate of the stable signal, the total rate of recognition was not good enough for the use of disables with unstable ratio signals. Hsieh et al. [6] have proposed an adaptive variable-ratio threshold prediction (AVRTP) algorithm to improve the recognition rate of the Morse code automatic recognition system. They also have successfully improved the situation to deal with the problem. However the AVRTP requires mass computation and great deal of computing time. Fuh \& Luo [7] have used a back propagation neural network (BPN) with the simplest network one neuron in input layer and one neuron in hidden layer to improve the recognition rate of the Morse code automatic recognition system. Since BPN is a nonlinear algorithm, it is too complicated to implement in single chip for online learning and recognition.

This paper proposed another new method of online learning and pattern recognition, which is the Expert-Gating neural network model with a linear Perceptron algorithm to shorten the computing time for online signals learning and recognition. In order to implement an algorithm to a single chip, we also presented a linear back propagation recalling algorithm.

Recently, neural networks have been used in pattern recognition problem, especially where input patterns are scale changed and shifted in position. Jacobs and Jordan [8] have designed a modular neural network to perform control tasks. Gallant [9] has presented Perceptron-based learning algorithm. These algorithms are suitable for applications in machine learning, pattern recognition, and connectionist expert system. Mitra and $\mathrm{Pal}$ [10] have proposed Fuzzy-MultiLayer Perceptron algorithm (A connectionist expert system model). Which is test on the speech recognition problem, on same medical data and on artificially generated intractable (linearly non-separable) pattern classes. Chen et al. [11] have used a modified HME
Architecture for text-dependent speaker identification. This algorithm used S-gating to enhance the results accompanied with those feature frames of the speaker's related information. Geva [12] has proposed a Multiscale neural network with multi-layer Perceptron to predict time series. Souheil et al. [13] have presented Combining experts used in binary classification face and speech data. Gutta et al. [14] described the application of mixtures of experts on gender and ethnic classification of human faces, and pose classification. Rahman et al. [15] presented a multiple-expert framework for recognition of handwritten characters. Almost of all researches [8-15] used multi-dimension signals and multi-layer or nonlinear network to classify patterns more precisely. Those algorithms could not easily and quickly be simulated and implemented in single chip. Algorithms with linear Perceptron or linear BPN could be simply and easily implemented in single chip. This linear operation on input vector is more efficient in computation compared to computing Euclidean distances. Its hardware implementation is simple and efficient also.

\section{METHOD}

Expert-Gating neural network model consists of tone recognition network (Expert) and silent recognition network (Gating). In this study, we use Expert neural network to recognize Morse-code tone time series and Gating neural network to deal with silent time series, which namely is dot (dot-space) or dash (dashspace). Figure 1 shows Mores code recognition system. The symbol $E_{l}(n)$ denotes the nth number of Morse code tone time series and $G_{s}(n)$ denotes the nth number of Morse code silent time series at the input layer. Expert network recognized tone time series with binary Perceptron algorithm and the anti-symmetric threshold function to decide output $Y,(n)$ is either dot $(-1)$ or dash $(+1)$. Gating network recognized silent time series with binary Perceptron algorithm and the step threshold function to decide $Y_{s}(n)$ is either dash-

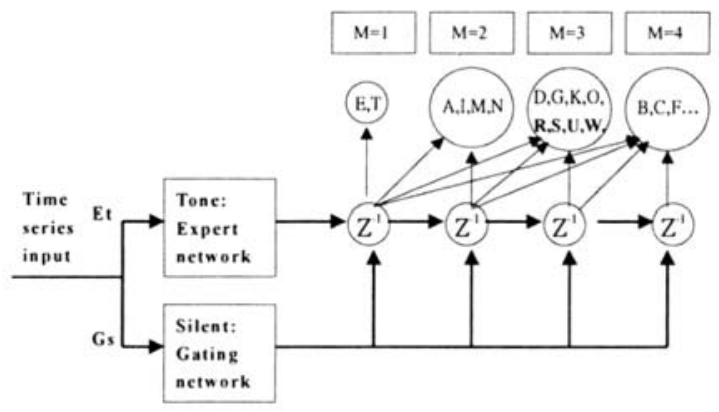

Fig. I Expert-Gating neural network model Morse code online recognition system. 
space $(+1)$ or dot-space (zero). When Gating network output is dot-space (zero), Expert network feeds its output signal to next memory register neuron and takes down $M=M+1(M$ : indicates the group of characters.). Gating network output is used to stimulate memory register neuron, when its output value is equal to one. After Expert neural network recognized, if Gating neural network output was equal one, then memory register was mapped to character classify. If $M$ equals to one, only the first group of characters are classified; if $M$ equals to two, then the second group of characters are classified, and so forth.

\subsection{Expert and Gating algorithm:}

Figure 2 shows the Expert time series recognition system. Input the nth number of tone time series $E_{t}(n)$, use previously threshold $E_{\theta}(n-1)$ to predict and calculate current threshold value $E_{\theta}(n)$, also, use binary Perceptron algorithm and anti-symmetric threshold function to determine whether $Y_{t}(n)$ is either $\operatorname{dot}(-1)$ or dash $(+1)$. We thus write:

$E_{\theta}(n)=E_{\theta}(n-1)+\eta_{1}\left[E_{1}(n) w_{1}(n)-E_{\theta}(n-1)\right]$

With anti-symmetric threshold function to determine that

If $E_{f}(n) w_{1}(n) \leq E_{\theta}(n)$, then the nth number of the tone time series is belonging to dot, i.e. $Y(n)=-1$

If $E_{t}(n) w_{t}(n)>E_{\theta}(n)$, then the nth number of the tone time series is belonging to dash, i.e. $Y_{t}(n)=+1$

$\left(\eta_{t} ;\right.$ indicates tone signal predict learning parameter. $w_{1}(n)$ : indicates tone signal synaptic weight. $n:$ indicates the input number of tone signal.)

Figure 3 shows the Gating time series recognition system. Input the nth number of silent time series $G_{s}(n)$, use previously threshold $G_{\theta}(n-1)$ to predict and calculate current threshold value $G_{\theta}(n)$, also use binary Perceptron algorithm and step threshold function (hard limiter) to determine whether $Y_{s}(n)$ is either dash-space $(+1)$ or dot-space (zero). That is:

$G_{\theta}(n)=G_{\theta}(n-1)+\eta_{s}\left[G_{s}(n) w_{s}(n)-G_{s}(n-1)\right](4)$

Then use step threshold function to determine that:

If $G_{s}(n) w_{s}(n) \leq G_{\theta}(n)$, then the nth number of the silent time series is belonging to dot-space, i.e.

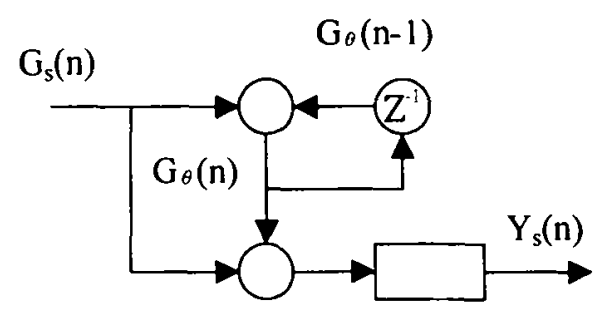

Step function

Fig. 3 Gating neural network (silent time series) recognition system.
$Y_{s}(n)=0$

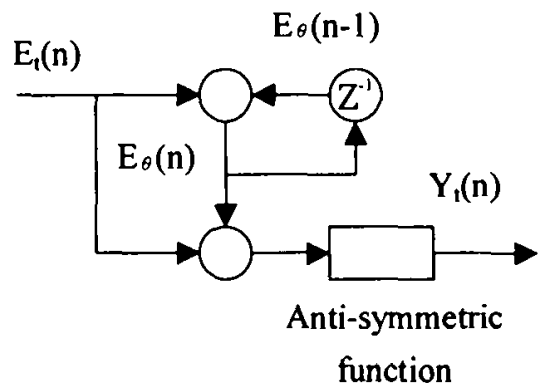

Fig. 2 Expert neural network (tone time series) recognition system.

If $G_{s}(n) w_{s}(n)>G_{\theta}(n)$, then the nth number of the silent time series is belonging to dash-space, i.e. $Y_{s}(n)=+1$

$\left(\eta_{s}:\right.$ indicates the predict learning parameter of silent signal. $w_{s}(n)$ : indicates the synaptic weight of silent signal. $n$ : indicates the input number of silent signal.)

\subsection{Integrate Expert algorithm and Gating algo-} rithm:

1. Set initial value $M$ equal to one, that is, it indicates the first group characters; and then clear all memory registers. Let tone time series $Y_{r}(n)$ be saved in the first memory neuron.

2. If the first silent time series $Y_{s}(n)$ is equal to one, then let the first tone time series $Y_{1}(n)$ in the first memory neuron go to map to the first group characters and classification, then go back to step 1 and classify next character. If $Y_{s}(n)$ is equal to zero, then $Y_{f}(n)$ in the first memory neuron would be delayed to the second memory neuron and let $M$ equal to two and go to step 3 .

3. Save the second tone time series into the first memory neuron. If the second silent time series $Y_{s}(n)$ is equal to one, then the first and the second tone time series $Y_{,}(n-1)$ and $Y_{t}(n)$ go to map to the second group characters, and then go back to step 1 and classify next character. If $Y_{s}(n)$ equals to zero, let $Y_{(}(n-1)$ delay to the third memory neuron and $Y_{,}(n)$ delay to the second memory neuron and set M equal to three and go to step 4.

4. Save the third tone time series into the first memory neuron. If the third silent time series $Y_{s}(n)$ equals to gne, then all the three tone time series $Y_{t}(n-2)$, $Y_{t}(n-1)$ and $Y_{i}(n)$ go to map to the third group characters, then go back to step 1 and classify next character. If $Y_{s}(n)$ equals to zero, let $Y_{i}(n-2)$ delay to the fourth memory neuron, $Y(n-1)$ delay to the third memory neuron and $Y_{i}(n)$ delay to the second memory neuron and set $M$ equal to four and go to next step.

5. Save the new tone time series into the first memory 
neuron. If the fourth silent time series $Y_{s}(n)$ equals to one, then let all the four tone time series $Y_{I}(n-3), Y_{I}(n-2), Y_{,}(n-1)$ and $Y_{i}(n)$ go to map to the fourth group characters, then go back to step 1 and classify next character. If $Y_{s}(n)$ equals to zero, it is then a mistake, since Morse code have only four codes in 26 letters of the alphabet (AB...Z). It can only continue to the next step, if auto recognized system has the above four Morse code characters.

\subsection{Linear BPN recalling}

This method uses computer learning and online recognized on a single chip. BPN algorithm learning system [7] is used to get average synaptic weights $\left(w_{l}(n), w_{\mathrm{K}}(n)\right)$ and average thresholds $\left(\theta_{k}, \theta_{j}\right)$. Initial synaptic weights $w_{1 \prime}(0)$ and $w_{j \mathrm{~K}}(0)$ are random numbers. Let learning parameter $\eta$ is between $0.5 \sim 5$. Used $f(v(n))=1 /[1+\exp (-v(n))]$ as output layer and hidden layer active functions. The symbols are defined as follows: $y_{1}(n)$ denotes an input layer signal; $y_{j}(n)$, denotes a hidden layer response; $y_{k}(n)$, denotes an output layer response; $\theta_{j}$, a hidden layer threshold; $\theta_{k}$, an output layer threshold.

Original recalling equation is shown as follow: 1. Input a pattern and use the equation shown below to compute the response of the hidden neuron.

$$
\begin{aligned}
& v_{,}(n)=w_{1 j}(n) y_{i}(n)-\theta, \\
& y_{i}(n)=f_{j}\left(v_{j}(n)\right)
\end{aligned}
$$

2. Use the following equation to compute the response of the output layer:
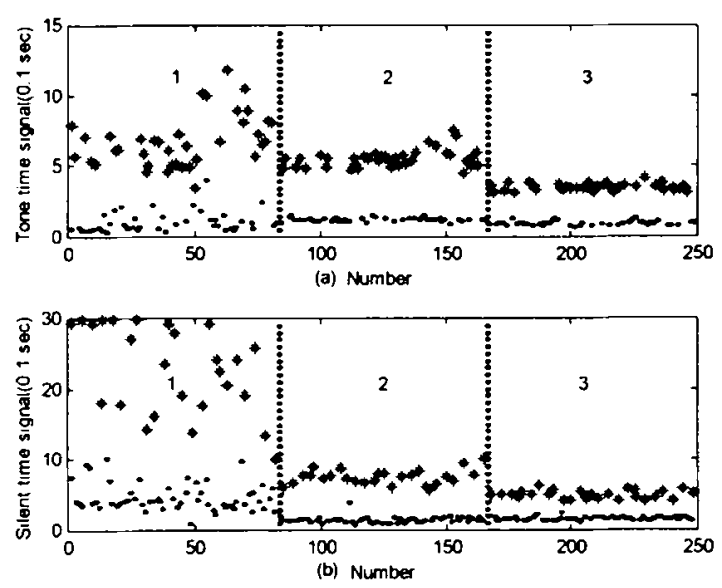

Fig. 4. Morse code pattern distributions: (a) Tone time signals, (b) Silent time signals.

Zone 1 displays the data from the cerebral palsy.

Zone 2 displays the data from the beginner.

Zone 3 displays the data from the skilled user.

'*' indicates dash, ' $\bullet$ ' indicates dot.

$$
\begin{aligned}
& v_{k}(n)=w_{j k}(n) y_{j}(n)-\theta_{k} \\
& y_{k}(n)=f_{k}\left(v_{k}(n)\right)
\end{aligned}
$$

Decision rule:

Since $y_{k}(n)=f_{k}\left(v_{k}(n)\right)=1 /\left[1+\exp \left(-v_{k}(n)\right)\right]$, if output $y_{k}(n)$ equal 0.5 , then $v_{k}(n)=0$, and then threshold $\theta_{k}$ equals to hidden synaptic weight $\left(w_{i j}(n)\right)$ product hidden layer output $y_{j}(n)$, therefore $\theta_{k}=w_{j k}(n) y_{j}(n)$. Use this condition to guide linear BPN recalling process. Which is shown by:

$$
y_{i}(n)=\frac{\theta_{j}-\ln \left(\frac{w_{y}(n)}{\theta_{k}}-1\right)}{w_{1 j}(n)}
$$

In formula (9), $w_{i j}(n), w_{j k}(n), \theta_{k}, \theta_{1}$ can be received from BPN learning process, so $y_{1}(n)$ is a constant. Therefore, if input signal is larger than $y_{1}(n)$, it will be decided as a dash (or dash-space); if the signal is less than $y_{1}(n)$, it will be taken as a dot (or dot-space). When this simplified process is implemented in a single chip as an online automatic recognition system, it would be very easy.

\section{EXPERIMENTAL RESULTS}

\section{Expert-Gating algorithm experiment:}

We used three persons to test in this experiment. Each person recognizes 26 letters of the alphabet (AB...Z). TABLE I shows the results of typing rate: Test one is a cerebral palsy, whose average typing rate is 14.99 characters per minute, Test two is a beginner, with average typing rate of 25.7 characters per minute, Test three is an expert, with average typing rate of 43.4 characters per minute. Figure 4 shows three tests of Morse code time series distribution. In which, Zone 1 displays the data from the cerebral palsy case, Zone 2 from the beginner user and Zone 3 from the skilled user. In zone 1, it has more dispersed time series. In zone 2 and 3, the dot (dot-space) is more stable than dash (dash-space). At the same time, we found some characters were mistyped and their corresponding Morse codes were mislabeled. The distribution of tone signals and silent signals are similar, therefore, we let tone predict learning parameters and correct learning parameters the same as silent.

In the testing process, the initial values of thresholds are used with two methods, one is inputting 6 characters Morse code $(e \cdot t \cdot i \cdot a \cdot n \cdot m$, i.e. $[\cdot,-$ $, \cdot \bullet,--,-\bullet,--$ ], ' $\bullet$ ' denotes the dot time series and '-' denotes the dash time series), which have ten tone signals and ten silent signals. Then, take the average value of tone signals and the average value of silent signals as initial thresholds $\left(E_{\theta}(0)\right.$ or $\left.G_{\theta}(0)\right)$. The second method is the use of $\mathrm{AB} \ldots \mathrm{Z}, 26$ letters of 


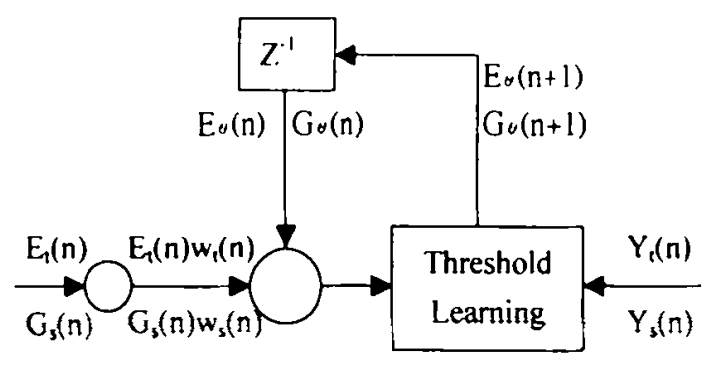

Fig. 5 Morse code initial thresholds learning system.

the alphabet, for online supervised learning to get initial tone time series threshold and silent time series threshold $\left(E_{\theta}(0)\right.$ or $G_{\theta}(0)$ ). Figure 5 shows Morse code initial threshold learning system. Let synaptic weights $w_{t}(n)$ and $w_{s}(n)$ are equal one. Correct learning rate $\left(\eta_{11}, \eta_{l}\right)$ are between $0.5-4$. Pruning input outline signals, if time series are shorter than 0.05 second, then we set it as 0.05 second; if time series are longer then three second, we let it be three seconds.

\section{Expert-gating neural network supervised learning correct rule:}

Input the nth number of tone signal and correct the threshold $E_{\theta}(n+1)$.

If $Y_{1}(n)=-1$ (dot) and $E_{1}(n) w,(n)-E_{\theta}(n)>0$,

Or

If $Y_{1}(n)=+1$ (dash) and $E_{1}(n) w_{1}(n)-E_{\theta}(n) \leq 0$,

Then correct the tone signal threshold value as:

$E_{\theta}(n+1)=E_{\theta}(n)+\eta_{t}\left[E_{t}(n) w_{t}(n)-E_{\theta}(n)\right]$

Input the nth number of silent signal and correct the threshold $G_{\theta}(n+1)$.

If $Y,(n)=0$ (dot-space) and

$G_{,}(n) w_{,}(n)-G_{e}(n)>0$,

Or

If $Y_{,}(n)=+1$ (dash-space) and

$G,(n) w_{s}(n)-G_{\theta}(n) \leq 0$,

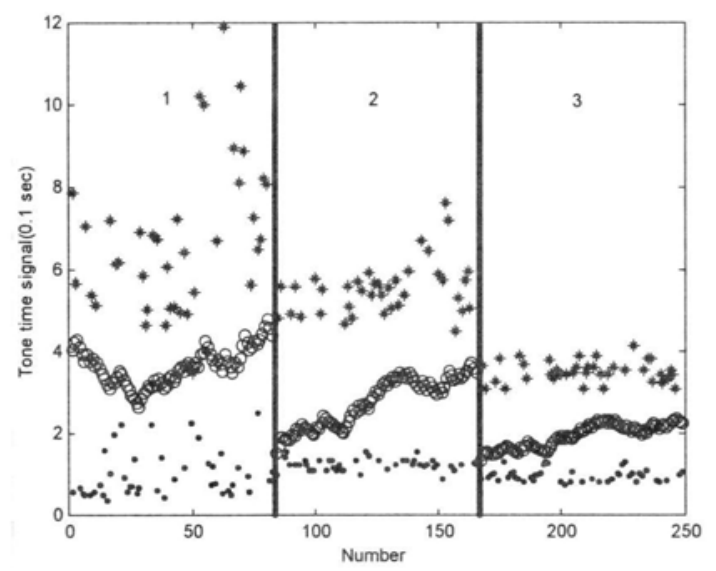

Fig. 6 Shows the figure $4($ a) tone signals predict learning the trajectory of thresholds on recognition process, correct learning parameter $\eta_{l t}$ and $\eta_{l}$ are equal to 1.0 , and initial thresholds track learning parameter $\eta_{\text {c }}$ and $\eta$, are equal to 0.05 . (' $*$ ' indicates dash, ' - ' indicates dot, ' 0 ' indicates threshold.)

Then correct the silent signal threshold value as: $G_{\theta}(n+1)=G_{\theta}(n)+\eta_{h}\left[G(n) w_{,}(n)-G_{\theta}(n)\right]$

Figure 6 . Shows the figure $4(a)$ tone signals the trajectory of thresholds on recognition process. Where the correct learning parameters $\left(\eta_{11}, \eta_{i s}\right)$ are all equal to 1.0 , and predict learning parameters $\left(\eta, \eta_{s}\right)$ are all equal to 0.05 (" indicates dash, ' $\bullet$ ' indicates dot, ' 0 ' indicates threshold.).

TABLE II shows the results of three tests recognition rate. Initial threshold values are the average of 6 characters. Tone signals predict leaming parameter $\eta$, and silent signals predict learning parameter $\eta_{\text {. }}$ are set between $0.5 \sim 0.01$. From the experiment of test one, we have $91.15 \%$ online average recognition rate; test two with $95.77 \%$, test three with $98.46 \%$ respectively. The improved predict learning parameters are nearly about 0.05 . TABLE III $\sim V$ shows the results of

TABLE I Shows the results of three tests average typing rate per minute.

\begin{tabular}{|c|c|c|c|}
\hline Typing rate & Test one & Test two & Test three \\
\hline 1 & 12.56 & 24.81 & 35.20 \\
\hline 2 & 12.65 & 24.87 & 38.86 \\
\hline 3 & 13.46 & 24.89 & 39.11 \\
\hline 4 & 14.68 & 24.94 & 41.05 \\
\hline 5 & 15.36 & 25.60 & 44.35 \\
\hline 6 & 15.63 & 25.67 & 44.75 \\
\hline 7 & 15.87 & 25.75 & 44.90 \\
\hline 8 & 16.20 & 25.86 & 45.45 \\
\hline 9 & 16.61 & 26.51 & 48.86 \\
\hline 10 & 16.92 & 28.08 & 51.44 \\
\hline Average & 14.99 & 25.70 & 43.40 \\
\hline
\end{tabular}


three tests, initial thresholds are used 26 characters online learning one epoch. TABLE III shows the result of recognition rates from test one, TABLE IV shows the results of test two and TABLE $V$ shows the results of test three. Set the correct learning rate $\left(\eta_{l}=\eta_{s}\right)$ between $0.5 \sim 4$, predict learning rate $\left(\eta_{t}=\eta_{s}\right)$ set between $0.01 \sim 0.5$. Symbol '*' in the table indicates that the recognition rate is lower than $50 \%$. TABLE III shows that the highest recognition rate is about $91 \%$, with correct learning parameter between $0.5 \sim 3$ and predict learning parameter between $0.1 \sim 0.01$. TA-
BLE IV shows that the highest recognition rate is about $96 \%$, with correct learning parameter between $0.5 \sim 3$ and predict learning parameter between $0.05 \sim 0.01$. TABI.E $V$ shows that the highest recognition rate is about $99 \%$, with correct learning parameter between $0.5 \sim 3$ and predict learning parameter between $0.1 \sim 0.01$. From the experiment of three tests, we found that correct learning parameters are not important factor for recognition rate.

\subsection{Linear BPN recalling experiment:}

TABLE II Three tests recognition rate, initial threshold values are the average value of 6 characters (e * $\mathbf{t} \cdot \mathbf{i} \cdot \mathbf{a} \cdot \mathbf{n} \cdot \mathbf{m})$.

\begin{tabular}{|c|c|c|c|c|c|}
\hline $\begin{array}{c}\text { Predict } \\
\text { Learning rate }\end{array}$ & 0.5 & 0.3 & 0.1 & 0.05 & 0.01 \\
\hline Test one & 67.31 & $\mathbf{8 0 . 3 8}$ & 90.77 & 91.15 & 90.38 \\
\hline Test two & $\mathbf{8 8 . 4 6}$ & 93.85 & 95.0 & 95.77 & 95.77 \\
\hline Test three & 91.54 & 95.0 & 98.46 & 98.46 & 96.15 \\
\hline
\end{tabular}

TABLE III Test one recognition rate, initial threshold values are correct learning 26 characters. $\left(\eta_{t q}=\eta_{t s}\right.$, $\eta_{t}=\eta_{s}$ ) Symbol '*' indicates that the recognition rate is lower than $50 \%$.

\begin{tabular}{|c|c|c|c|c|c|c|}
\hline$\eta_{l}=\eta_{\text {is }}$ & 0.5 & 1.0 & 1.5 & 2.0 & 3.0 & 4.0 \\
\hline 0.5 & 67.31 & 67.31 & 67.31 & 67.31 & 67.31 & 58.85 \\
\hline 0.3 & 80.38 & 80.38 & 80.38 & 80.38 & 80.38 & 60.77 \\
\hline 0.1 & 90.77 & 90.77 & 90.38 & 90.77 & 90.77 & $*$ \\
\hline 0.05 & 91.54 & 91.54 & 91.54 & 91.54 & 91.54 & $*$ \\
\hline 0.01 & 90.38 & 90.38 & 90.77 & 91.54 & 90.38 & $*$ \\
\hline
\end{tabular}

TABLE IV Test two recognition rate, initial threshold values are correct learning 26 characters. $\left(\eta_{h t}=\eta_{l s}\right.$, $\eta_{1}=\eta_{\mathrm{s}}$ ) Symbol '*' indicates that the recognition rate is lower than $50 \%$.

\begin{tabular}{|c|c|c|c|c|c|c|}
\hline$\eta_{L}=\eta_{k}=\eta_{k}$ & 0.5 & 1.0 & 1.5 & 2.0 & 3.0 & 4.0 \\
\hline 0.5 & 88.46 & 88.46 & 88.46 & 88.46 & 88.46 & 87.69 \\
\hline 0.3 & 93.85 & 93.85 & 93.85 & 93.85 & 93.85 & 90.77 \\
\hline 0.1 & 95.0 & 95.0 & 95.0 & 95.0 & 95.0 & 76.54 \\
\hline 0.05 & 96.15 & 96.15 & 96.15 & 96.15 & 96.15 & 69.62 \\
\hline 0.01 & 96.15 & 96.15 & 96.15 & 95.0 & 95.0 & $*$ \\
\hline
\end{tabular}

TABLE $V$ Test three recognition rate, initial threshold values are correct learning 26 characters. $\left(\eta_{k t}=\eta_{l s}\right.$, $\eta_{t}=\eta_{s}$ ) Symbol '*' indicates that the recognition rate is lower than $50 \%$.

\begin{tabular}{|c|c|c|c|c|c|c|}
\hline$\eta_{L}=\eta_{l s}$ & 0.5 & 1.0 & 1.5 & 2.0 & 3.0 & 4.0 \\
\hline 0.5 & 91.54 & 91.54 & 91.54 & 91.54 & 91.54 & 88.46 \\
\hline 0.3 & 98.85 & 98.85 & 98.85 & 98.85 & 98.85 & 90.77 \\
\hline 0.1 & 99.23 & 99.23 & 99.23 & 99.23 & 99.23 & 80.38 \\
\hline 0.05 & 99.23 & 99.23 & 99.23 & 99.23 & 99.23 & $*$ \\
\hline 0.01 & 99.23 & 98.85 & 98.85 & 98.85 & 98.85 & $*$ \\
\hline
\end{tabular}


TABLE VI Morse code recognition rates from three tests by three methods: linear BPN recalling method (LBPN); 6 characters average value as initial thresholds (EG6) and 26 characters learning as initial thresholds (EG26).

LBPN: Learning rate $\eta$ ) equal to 2, and a learning cycle of ten epochs.

EG6: Predict learning rate equal to 0.05 .

EG26: Correct learning rate equal to 1.0 , predict learning rate equal to 0.05 .

\begin{tabular}{|l|l|l|l|l|l|l|l|l|l|}
\hline & \multicolumn{3}{|l|}{ Test one } & \multicolumn{3}{l|}{ Test two } & \multicolumn{3}{l|}{ Test three } \\
\hline Method & LBPN & EG6 & EG26 & LBPN & EG6 & EG26 & LBPN & EG6 & EG26 \\
\hline Average & $\mathbf{9 2 . 3}$ & $\mathbf{9 1 . 2}$ & $\mathbf{9 1 . 5}$ & $\mathbf{9 6 . 2}$ & $\mathbf{9 5 . 8}$ & $\mathbf{9 6 . 2}$ & $\mathbf{9 9 . 2}$ & $\mathbf{9 8 . 5}$ & $\mathbf{9 9 . 2}$ \\
\hline
\end{tabular}

In this test, the linear BPN recalling algorithm uses Morse code AB...Z, 26 letters of the alphabet. For online supervised learning to get average synaptic weights and average thresholds $\left(w_{i j}(n) 、 w_{j k}(n) \cdot \theta_{k}\right.$, $\theta_{1}$ ), we set learning rate $(\eta)$ equal to 2 , and no decaying learning rate, momentum $\alpha$ equal to zero, and a learning cycle of ten epochs. Use equation (9) to calculate $y_{1}(n)$ as a threshold and classify Morse code.

TABLE VI shows the results of the three tests for three methods, where linear BPN recalling method (LBPN) with learning rate $(\eta)$ equal to 2 and a learning cycle of ten epochs. From the experiment of test one, we have $92.31 \%$ online average recognition rate, test two $96.15 \%$, test three $99.23 \%$ respectively.

\section{DISCUSSION AND CONCLUSIONS}

Figure 4 shows that pattern scale and distribution are different in three tests. Test one has the largest scale than test two and test three. It also has more mistypes and Morse code overlap. Patterns have different scale and distribution means that the tone rate and silent rate are different. Therefore, the optimal correct learning rates $\left(\eta_{l l}, \eta_{l s}\right)$ may be different in each test. In this experiment, correct learning rates is between $0.5 \sim 4$. From our experiment, we knew that correct learning parameter is not the major factor for recognition rates. The Morse code distribution also reveals that dot is more stable than dash. Which means that predict learning rate may differ in dot and dash on each test. The predict learning rate from 0.01 to 0.5 are taken in the test and we have experienced that 0.05 is the better one for all three tests.

Since Morse code is a one-dimensional time series signals, therefore, linear Expert-Gating neural network model and linear BPN recalling algorithm both can solve the problem of recognition with very short learning and classified time. Usually, neural network requires mass computation and great deal of computing time. To shorten the computing time for online signal recognition, we can implement linear Expert-Gating neural network algorithm in single chip with one cycles of learning and obtain satisfactory recognition results.

Through signals analysis, we found that some characters were mistyped and their corresponding Morse codes were mislabeled. That is why the recognized rate couldn't reach up to $100 \%$.

For further studies, there are still some problems need to be solved. First, the signal should be appropriately preprocessed before inputting it. Second, we have to choose the simplest neural network. We also can use other kinds of neural net, such as, linear SelfOrganizing-Map or neural fuzzy, to solve the more complex problem.

\section{Acknowledgement}

This work is supported by the grants NSC 902614-E-006-004 from National Sciences Council, TAIWAN.

\section{REFERENCES}

1. Arnott JL and Javed MY: Probabilistic character disambiguation for reduced keyboards using small text samples. AAC Augmentative and Alternative Communication 1992; 8: 215-223.

2. Higginbotham DJ: Evaluation of keystroke savings across five assistive communication technologies. AAC Augmentative and Alternative Communication 1992; 8: 258-272.

3. Hauck LT: SAM: An improved input device. Johns Hopkins APL Technical Digest 1992; 13: 490-493.

4. Luo $\mathrm{C}-\mathrm{H}$ and Shih $\mathrm{C}-\mathrm{H}$ : Adaptive Morse-coded single-switch communication system for the disabled. Int. J. Bio-Medical Computing 1996; 41: 99 106.

5. Shih $\mathrm{C}-\mathrm{H}$ and Luo $\mathrm{C}-\mathrm{H}$ : A Morse-code recognition system with LMS and matching algorithms for persons with disabilities. Int. J. Medical Informatics 1997; 44: 193-202.

6. Hsieh $\mathrm{M}-\mathrm{C}$, Luo $\mathrm{C}-\mathrm{H}$ and Mao C-W: Unstable Morse Code Recognition with Adaptive Variable Ratio Threshold Prediction for Physically Disabled Persons. IEEE Trans. on Rehabilitation Engineering 2000; 8: 405-413.

7. Fuh D-T and Luo C-H: Unstable Morse-code auto recognition system with back propagation neural 
network for person with disabilities. Journal of Medical Engineering \& Technology 2001; 25: 118123.

8. Jacobs RA and M.I. Jordan MI: Learning piecewise control strategies in a Expert-Gating neural network architecture. IEEE Transactions on Systems, Man, and Cybernetics 1993; 23: 337-345.

9. Gallant SI: Perceptron-Based Learning Algorithms. IEEE Transactions on neural networks 1990; 1: 179-191.

10. Mitra S and Pal SK: Fuzzy Multi-Layer Perceptron, Inferencing and Rule Generation. IEEE Transactions on neural networks 1995; 6: 51-63.

11. Chen K, Xie D and Chi H: A Modified HME Architecture for Text-Dependent Speaker Identification. IEEE Transactions on neural networks 1996; 7: 1309-1313.
12. Geva AB: ScaleNet Multiscale Neural Network Architecture for Time Series Prediction. IEEE Transactions on neural networks 1998; 9: 14711482.

13. Souheil BY, Yousri A and Eddy M: Fusion of Face and Speech Data for Person Identity Verification. IEEE Transactions on neural networks 1999; 10: 1065-1074.

14. Gutta S, Huang RJJ, Jonathon $P$ and Wechsler $H$ : Mixture of Experts for Classification of Gender, Ethnic Origin, and Pose of Human Faces. IEEE Transactions on neural networks 2000; 11: 948-960.

15. Rahman AFR, Howells WGJ, and Fairhurst MC: A Multiexpert Framework for Character Recognition: A Novel Application of Clifford Networks. IEEE Transactions on neural networks 2001; 12: 101-112. 